\title{
Thermoelastic study of an aluminum component using an automatic correction procedure of data acquired in non-adiabatic conditions
}

\author{
by A. Gallotti, S. Desiderati and A. Salerno
}

Dipartimento di Energia, Politecnico di Milano, via Lambruschini 4, 20156 Milano, Italy antonio.salerno@polimi.it

\begin{abstract}
Quantitative results in Thermoelastic Stress Analysis (TSA) are often affected by a systematic error, when the test is not performed in adiabatic conditions. A correction procedure can be implemented in order to recover the adiabatic temperature, i.e. the temperature distribution that would have been present if adiabatic conditions had been reached. Such correction procedure however depends on the right assumption on the kind of stress distribution, e.g. linear, parabolic or cubic. The most adequate stress distribution can be chosen automatically by performing the thermoelastic test at different frequencies. This technique allowed obtaining quantitative TSA results on a helicopter aluminum component.
\end{abstract}

\section{Introduction}

When a solid is subject to a sufficiently fast deformation and volume variation, produced by applying a load, it undergoes an adiabatic transformation with a consequent temperature variation. Thermoelastic Stress Analysis (TSA) is capable of linking such temperature variation to the variation of the first stress invariant in case of adiabatic conditions [1].

TSA is normally performed by applying a load, whose intensity is sinusoidally modulated in time. The result of a TSA measurement is one amplitude image, in which every point represents the amplitude of the modulated temperature, and one phase image, in which every point represents the phase shift of the modulated temperature with respect to the modulated load applied.

TSA tests on real metallic components seldom reach adiabatic conditions, because the load cannot be applied at a sufficiently high frequency, so as to prevent the heat diffusion from points assuming different temperatures during the modulated application of the load. This produces an attenuation of the temperature peaks and a systematic error in TSA results.

When adiabatic conditions are reached, the temperature variation is in phase or antiphase with the load applied, i.e. $\varphi=0^{\circ}$ or $180^{\circ}$ depending on the fact that a region can be under traction or compression.

When adiabatic conditions are not reached, the temperature amplitude of points having high stress concentrations undergo a temperature attenuation. Furthermore, in non-adiabatic conditions the phase shift between the load applied and the modulated surface temperature is different from $0^{\circ}$ or $180^{\circ}$ and it varies with the frequency, approaching these values when the modulation frequency of the load increases.

The analytical solution of the direct problem allowed to discover that the function linking the attenuation to the phase shift (correction function) only depends on the kind of stress distribution, for example linear, quadratic or cubic [2].

Based on the geometry, the constraints and the load applied, an operator can make an assumption on the kind of stress distribution and choose the more appropriate correction function.

For instance, for a cantilever beam fixed at one end and with a force normal to its axis applied to the other end, the stress distribution is linear, while near a hole in a beam subject to an axial load the stress distribution is more similar to a parabolic one.

Starting from the phase shift, the correction function gives the attenuation. Once the attenuation is known, from the measured temperature amplitude $\Delta T$ the adiabatic temperature amplitude $\Delta T_{\text {ad }}$ can be reckoned, which in turn is liked to the amplitude of the first stress invariant $\Delta \sigma$, determinable from the temperature through the thermoelastic equations.

Such a correction procedure was applied on several real components and gave results in good agreement with experimental data acquired by strain gauges, when available $[3,4]$.

However, applying the same correction function to every point of a component surface may be wrong. For instance, let us consider a cantilever beam with a hole in the center subject to a bending moment. Close to the hole edge the stress distribution could be approximated by a parabolic function, while far from the hole the dominant stress distribution is linear. Applying a parabolic or a linear correction on the whole image, would inevitably lead to a wrong correction on a portion of the image.

For some complicated geometries or for components on which more than one load is applied, choosing the more appropriate correction function could be not straightforward.

\section{The thermoelastic effect}

The thermoelastic effect was firstly studied by Kelvin [5] in 1853, but the basic equation relating the temperature variation $\delta \mathrm{T}$ to the first stress invariant variation $\delta \sigma=\sigma_{l}+\sigma_{I I}+\sigma_{I I I}$ was introduced by Darken and Curry [6] in 1953: 


\subsection{1/qirt.2016.113}

$$
\frac{\delta T}{T_{0}}=-K_{0} \delta \sigma
$$

where $T_{0}$ is the average temperature (normally the ambient temperature) and $K_{0}$, named thermoelastic constant, is a material parameter comprising the coefficient of linear thermal expansion $\lambda$, the mass density $\rho$ and the specific heat at constant pressure $c_{p}$ :

$$
K_{0}=\frac{\lambda}{\rho c_{p}}
$$

Applying equ. (1) to the Fourier equation, it is easy to obtain the expression of the thermoelastic heat source:

$$
q=-\rho c_{v} K_{0} T_{0} \frac{\partial \sigma}{\partial t}
$$

From this basic theory it is evident that only stress variations can give a thermoelastic heat source. A sinusoidally modulated load producing a sinusoidally modulated stress distribution is then normally applied to a component in TSA tests.

\section{Automatic correction}

Given a modulated stress distribution in a component, thermoelastic heat sources are distributed like the stress. Heat is then diffused in the sample if adiabatic conditions are not reached. The direct heat conduction problem can be easily solved analytically or numerically, using the Fourier equation, to obtain the temperature variation at different frequencies and for different stress distributions. attenuation:

For every stress distribution a correction function can be obtained linking the phase shift to the temperature

$$
1-\frac{\Delta T}{\Delta T_{a d}}=f_{\sigma}(\Delta \varphi)
$$

Where the first member is the temperature attenuation and $f_{\sigma}$ is the correction function.

From equ. (4) the adiabatic temperature variation can be readily obtained:

$$
\Delta T_{a d}=\frac{\Delta T}{1-f_{\sigma}(\Delta \varphi)}
$$

If a TSA test can for example be performed at three different frequencies, starting from three different phase shifts $\Delta \varphi_{i}(\mathrm{i}=1,2,3)$, three different adiabatic temperature variations $\Delta T_{a d, \mathrm{i}}$ can be evaluated using the same correction function $f_{\sigma}$

The idea at the base of the automatic correction [7] is that if the real stress distribution corresponds to the stress distribution for which the direct Fourier problem has been solved, and the correspondent correction function determined, the reckoned adiabatic temperature variation $\Delta T_{a d}$ should be correct and independent from the frequency.

So for instance if the real stress distribution is linear, a correction function obtained for a linear stress distribution should give a correct value for the adiabatic temperature variation $\Delta T_{a d}$ regardless of the modulation frequency.

Since in reality the stress distribution for which the correction function has been determined does not perfectly correspond to the real stress distribution, performing $N$ tests at $N$ different frequencies, will give $N$ different values of the adiabatic temperature variation $\Delta T_{a d}$.

An average value: 
10.21611/qirt.2016.113

$$
\Delta T_{\text {ad-ave }}=\frac{1}{N} \sum_{i=1}^{N} \frac{\Delta T_{i}}{1-f_{\sigma}\left(\Delta \varphi_{i}\right)}
$$

and a standard deviation

$$
s t d=\sqrt{\frac{1}{N-1} \sum_{i=1}^{N}\left[\frac{\Delta T_{i}}{1-f_{\sigma}\left(\Delta \varphi_{i}\right)}-\Delta T_{a d-a v e}\right]^{2}}
$$

can therefore be obtained for a given correction function $f_{\sigma}$.

A correction function $f_{\sigma}$ corresponding to the real stress distribution would give always the same result, regardless of the frequency, and a zero value for the standard deviation $(s t d=0)$.

Many stress distributions can be considered and the same number of correction functions can be obtained analytically or numerically. Among them, a computer program can automatically select the correction function having the minimum value for the standard deviation, i.e. the correction function obtained starting from a stress distribution more closely corresponding to the real one.

The process is replicated for the available correction functions and for every pixel, so that for every pixel of the TSA amplitude image the best correction function is chosen.

The real correction of the amplitude TSA image is then performed pixel by pixel starting from the amplitude TSA image obtained at the highest available frequency, which, being closer to adiabatic conditions, is affected by the lower attenuation error.

\section{Component and experimental conditions}

The TSA study was performed on a helicopter metallic component comprising several parts in different aluminum alloys $(2024,7075,7475)$ riveted together.

The component was originally covered by a primer coating, which is always applied to aeronautic components to protect their surface. Such a primer coating was sufficiently thick to disturb the TSA measurement, introducing an additional attenuation and a phase shift also at low frequencies. The primer was then removed from the surface in the region of the measurement, to allow better conditions for obtaining quantitative thermoelastic results. The area of the TSA measurement was then spray painted in black so as to create a high emissivity paint coating, sufficiently thin not to disturb the thermoelastic measurements and avoid reflections from the environment.

The component had a platform on which an attachment was fixed with four bolts. A single hydraulic jack was acting on the component pulling the attachment. The platform was creating a shoulder, which locally complicated the shape and the stress distribution in the component.

Tests were performed using two different loads values at different frequencies according to table 1.

Table 1. Loads applied for the TSA test

\begin{tabular}{|l|l|l|l|l|l|}
\hline Load & Static [kg] & Dynamic [kg] & Max [kg] & Min [kg] & Freq. [Hz] \\
\hline A & 850 & 800 & 1650 & 50 & $1-3-6$ \\
\hline B & 1250 & 1200 & 2450 & 50 & $1-3$ \\
\hline
\end{tabular}

Load A had a dynamic component of $800 \mathrm{Kg}$ and it was applied at a maximum frequency of $6 \mathrm{~Hz}$.

Load $\mathrm{B}$ had a dynamic component of $1200 \mathrm{Kg}$, but the maximum possible frequency was only $3 \mathrm{~Hz}$.

Fig. 1 shows the area of interest near the attachment of the jack. One end of the attachment is visible and the shoulder formed by the platform on which the attachment was positioned. 


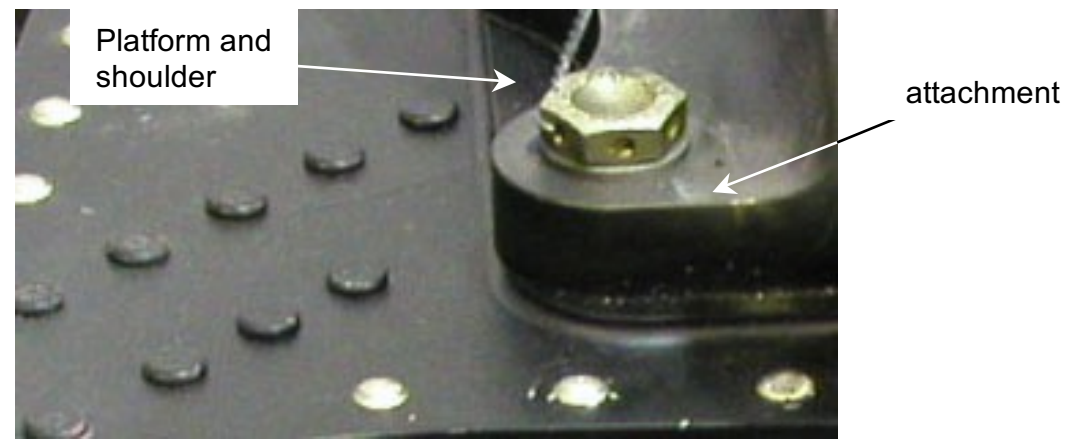

Fig. 1. Area of the attachment of the jack presenting a platform and a shoulder.

\section{TSA results and comparison with the results of a strain gauge rosette}

Fig. 2 shows the amplitude TSA image obtained with load A (800 kg dynamic) at $6 \mathrm{~Hz}$.

Fig. 3 shows the first stress invariant according to the TSA measurement along line $L$ of Fig. 2 applying load $A$ at three different frequencies: 1,3 and $6 \mathrm{~Hz}$. The non achievement of adiabatic conditions is evident from the rising of TSA stress with frequency.

At $1 \mathrm{~Hz}$ the maximum value is $15 \mathrm{MPa}$ with a strong attenuation. At $6 \mathrm{~Hz}$ the maximum value is approximately 27 $\mathrm{MPa}$, but adiabatic conditions are not yet reached and an attenuation is still present.

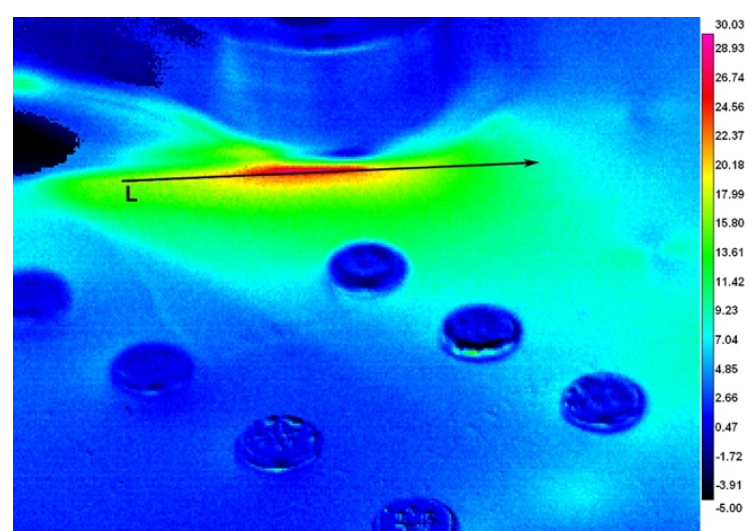

Fig. 2. TSA result obtained with load $A$ at $6 \mathrm{~Hz}$

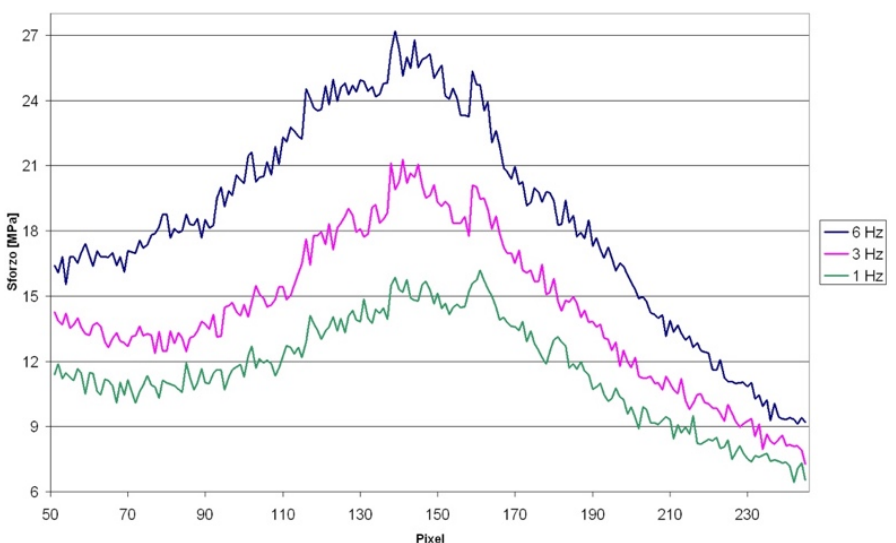

Fig. 3. The rising of the strain with frequency, evaluated using the thermoelastic relations, is a sign of non-adiabatic conditions

Fig. 4 shows the result of the automatic correction applied on the TSA amplitude image obtained with load A at 6 $\mathrm{Hz}$. The maximum value reaches $36 \mathrm{MPa}$ with a $33.33 \%$ increment in relation to the non corrected value of $27 \mathrm{MPa}$.

Comparing Fig. 2 and Fig. 4 and taking as a reference $20 \mathrm{MPa}$ (yellow colour on both images), one can easily observe the enlargement of the area stressed above this value.

The diagram of the stress along lines 1, 2 and 3 is shown in figures 5 to 7 . A steep stress gradient is present in the region. One of the advantages of TSA is that it produces a map of the stress distribution on the whole surface, easily allowing to spot the point of maximum stress concentration. 


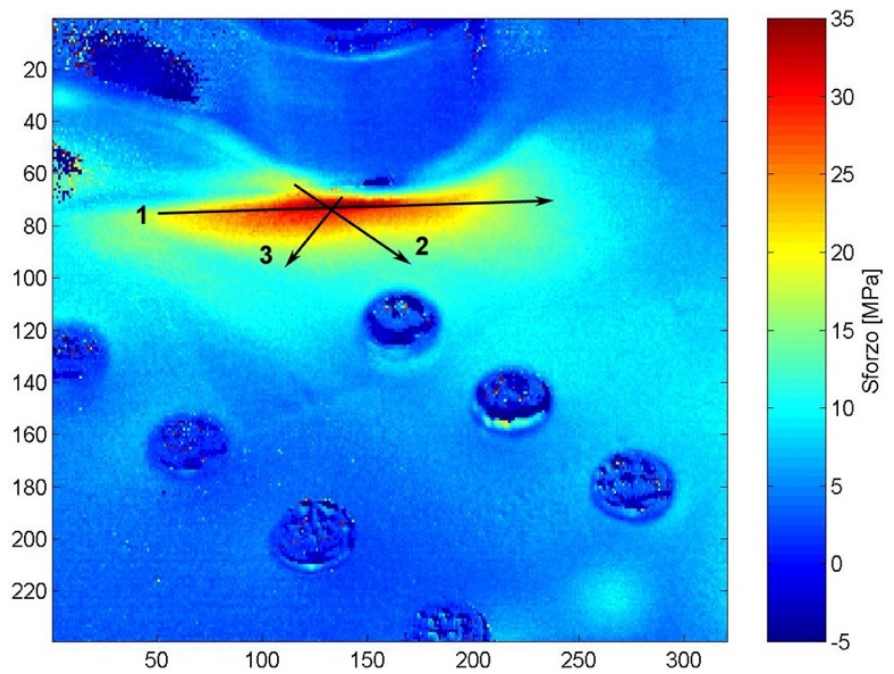

Fig. 4. Corrected stress values obtained applying the automatic correction on TSA amplitude images acquired with load $A$ at $6 \mathrm{~Hz}$.

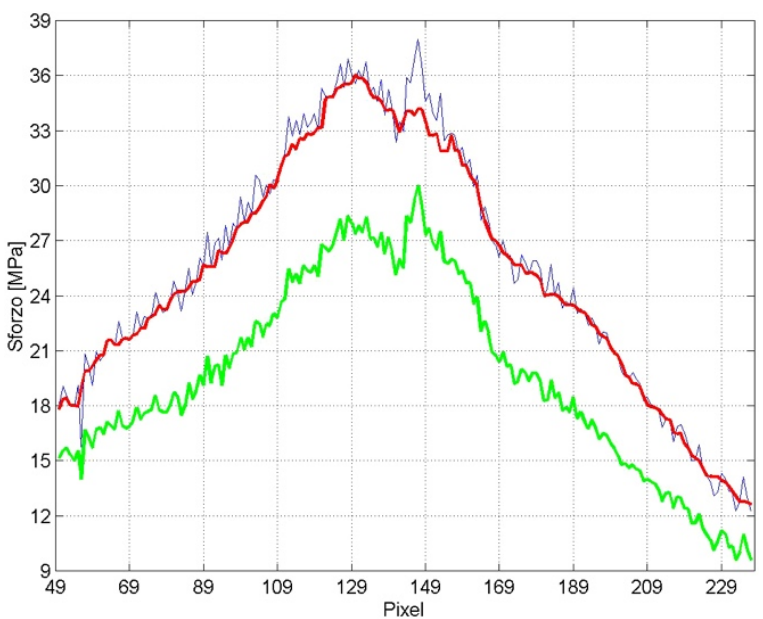

Fig. 2. Stress values along line 1of Fig. 4: non corrected (lower green line); corrected automatically (upper blue line); median filter of the corrected results (red line)

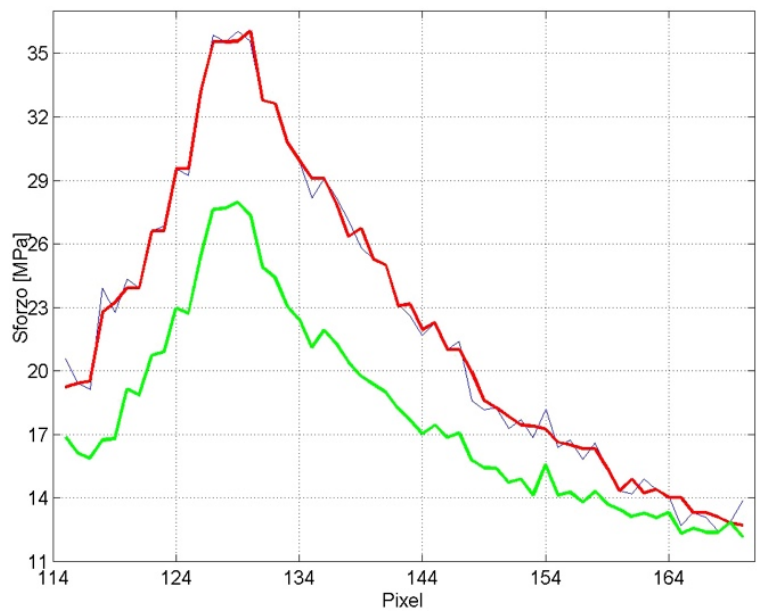

Fig. 3. Strain values along line 2 of Fig. 4: non corrected (lower green line); corrected automatically (upper blue line); median filter of the corrected results (red line)

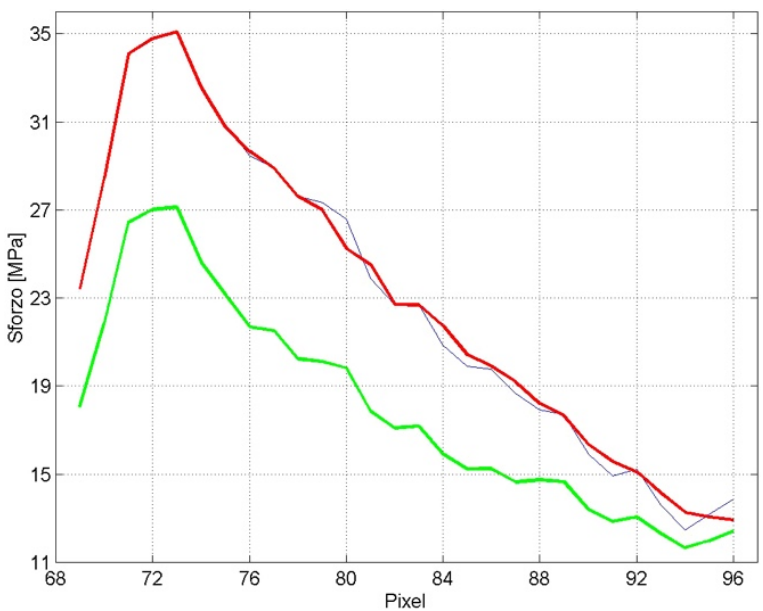

Fig. 4. Strain values along line 3 of Fig. 4: non corrected (lower green line); corrected automatically (upper blue line); median filter of the corrected results (red line) 
A strain gauge rosette was placed in a position near the attachment according to Fig. 8. The position of the strain gauge rosette did not correspond precisely to the observed peak of the first stress invariant.

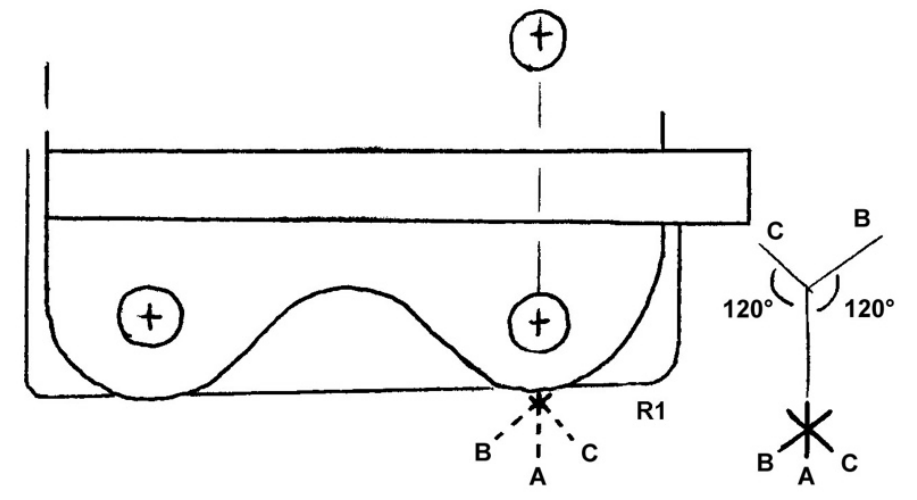

Fig. 5. Position of the strain gauge rosette on the component near the attachment.

While TSA can reveal the first stress invariant on the entire surface, a strain gauge rosette can measure the single directional components of the strain, but in a one point only. A further advantage of TSA is that it can read the first stress invariant with a very high spatial resolution, virtually limited only by the wavelength of the IR radiation used and by the quality of the optics and of the IR camera. On the contrary the result obtained by a strain gauge is the average deformation of an area equal to its surface, i.e. some millimeters.

TSA reads the peak to peak value of the first stress invariant, therefore, given a dynamic load of $800 \mathrm{Kg}$, the result must be compared with that of a strain gauge, when the component is statically loaded with $1600 \mathrm{Kg}$.

In this condition the first stress invariant measured by the strain gauge rosette was $28.93 \mathrm{MPa}$.

The precise position of the strain gauge rosette in relation to the stress distribution shown by the TSA test is difficult to determine, but the strain gauge rosette was placed in an area where the TSA gave a result comprised between 25 and $30 \mathrm{MPa}$.

\section{Conclusions}

TSA allows for the fast and contactless measurement of the first stress invariant distribution on a component under load. Real components cannot be loaded at high modulation frequencies and adiabatic conditions are almost never reached.

An automatic correction procedure was used to recover the adiabatic temperature starting from data acquired in non-adiabatic conditions. The adiabatic temperature was then converted into the first stress invariant (MPa) according to the thermoelastic equations. The results showed a local high stress concentration, whose maximum value reached 36 $\mathrm{MPa}$.

A strain gauge rosette was placed in a region where the TSA gave a result comprised between 25 and $30 \mathrm{MPa}$. The first stress invariant evaluated using the strain gauge rosette was $28.93 \mathrm{MPa}$.

\section{REFERENCES}

[1] Harwood N. and Cummings W. M., Thermoelastic Stress Analysis, IOP Publishing (Adam Hilger), Bristol, UK, 1991.

[2] Salerno A. and Desiderati S. "Procedure proposal for the correction of nonadiabatic thermoelastic stress analysis results", Review of Scientific Instruments, Vol. 75, N.2, pp. 507-514, 2004.

[3] D. Colombo, S. Desiderati, M. Giglio, A. Salerno, "TSA-FEM comparative analysis of a helicopter component under multiaxial load", Strain, Vol. 43, pp. 311-318, 2007

[4] S. Desiderati and A. Salerno, "Strain Gage Identification of the Correction Procedure for Quantitative Thermoelastic Stress Analysis under Non-adiabatic Conditions", Materials Evaluation, pp. 855-861, 2015

[5] Thomson W., "On the Dynamic Theory of Heat", trans. Royal Society, Vol. 20, pp. 261, 1853

[6] Darken L. and Curry R., Physical Chemistry of Metals, McGraw-Hill, London, 1953

[7] Gallotti A., Salerno A., "Automatic procedure for the correction of thermoelastic stress analysis data acquired in non-adiabatic conditions", Review of Scientific Instruments, Vol. 76, N. 12, pp. 124903-124903-7, 2005 